\title{
Structure Activity Studies on Chemically Modified Homologues of the Antibiotic Phytotoxic Leucinostatin A
}

\author{
G. Vertuani, M. Boggian and A. Scatturin
}

Dipartimento di Scienze Farmaceutiche, Università di Ferrara, I-44100 Ferrara, Italy

\author{
M. Ricci, B. Meli Balbocchino, L. Tuttobello and C. Rossi*
}

Istituto di Chimica Farmaceutica e Tecnica Farmaceutica, Università di Perugia, Via del Liceo 1, I-06100 Perugia, Italy

(Received for publication December 14, 1994)

\begin{abstract}
The synthesis and a conformational study of a number of homologues of the well known antibiotic, phytotoxic leucinostatin A are reported. The circular dichroism of all the compounds are discussed. Some conclusions on the SAR of these compounds are drawn. The influence of the $\alpha$-helical conformation and/or the increased lipophile character on their interesting biological activities is emphasized.
\end{abstract}

The nonapeptide leucinostatin $A$ is the major antibiotic, cytotoxic, phytotoxic component isolated, as hydrochloride salt, from cultures filtrates of Paecilomyces marquandii (Massee) Hughes. Additional minor metabolites leucinostatin $\mathrm{B}, \mathrm{C}, \mathrm{D}, \mathrm{F}, \mathrm{H}$ and $\mathrm{K}$ have been isolated from the same submerged cultures. The chemical structure of leucinostatin A has been worked out by several groups ${ }^{1 \sim 3}$. It turned out to be composed by the unsaturated fatty acid $(4 S, 2 E)$-4-methylhex-2-enoic acid at the $\mathrm{N}$-terminal; the $(2 S)-N^{1}, N^{1}$-dimethylpropane1,2-diamine at the C-terminal; and of the following nine amino acids: cis-4-methyl-L-proline, $(2 S, 4 S, 6 S)$-2-amino-6-hydroxy-4-methyl-8-oxodecanoic acid, threo- $\beta$ hydroxy-L-leucine, three $\alpha$-aminoisobutyric acids, two Lleucines and $\beta$-alanine. The composition of other leucinostatins is closely related ${ }^{4 \sim 7)}$ to that of leucinostatin A. All these co-metabolites of the phytopathogenic microorganism are endowed with a similar wide spectrum of antibacterial, antimycotic, cytotoxic, and phytotoxic activity. However, these biological activities are exhibited to a different extent by different leucinostatins ${ }^{2 \sim 7)}$. In particular, leucinostatin $\mathrm{D}$ is active against some strains of staphilococcus that are known to be resistant against more common antibiotics, and shows promising cytotoxic and phytotoxic activities ${ }^{5)}$. CD and IR spectroscopy studies on leucinostatin A hydrochloride have investigated the preferred conformations of the molecule in solvents of different polarity and at different concentrations ${ }^{8)}$. The results provide evidence that the peptide antibiotic, in apolar solvents and in the range of concentration used in the spectroscopic measurements, folds its backbone in a helical structure. Similar behaviour has been observed ${ }^{9)}$ in some synthetic fragments of leucinostatin $\mathrm{A}$ in lipophilic solvents, indicating a strong tendency to fold in an ordered structure. The crystal and molecular structure of the nonapeptide antibiotic has been also determined ${ }^{10}$ ). It was found that the peptide backbone folds in a regular right-handed $\alpha$-helix conformation with six intramolecular hydrogen bonds. In the crystal the helices are linked head to tail by hydrogen bonds and electrostatic interactions, forming continous helical rods.

Since the molecular structure of leucinostatin A is important in providing a basis for structure-activity studies and in obtaining an insight into the possible role of specific side chains and backbone folding in determining the biological activity, in this paper we report the results of a structure-activity study in solution carried out using $\mathrm{CD}$ and biological activity measurements of the nonapeptide homologues which have been obtained by chemical modification of native leucinostatin A.

\section{Materials and Methods}

Isolation of Leucinostatin A

Leucinostatin A hydrochloride was obtained from the benzene extract of the cultural broth by extensive flash chromatography fractionations on silica gel column in a nitrogen atmosphere. As eluent $\mathrm{CHCl}_{3}$ containing increasing amounts of $\mathrm{CH}_{3} \mathrm{OH}$, (up to $9 \%$ ) and of conc. $\mathrm{NH}_{4} \mathrm{OH}$ (up to $1 \%$ ) was used. After solvent evaporation the white material obtained was crystallized several times 
using dry acetone and ethyl acetate in order to obtain the separation between salt and free forms of leucinostatin A. Further details of isolation and purification of leucinostatin $\mathrm{A}$ are reported elsewhere ${ }^{11)}$.

\section{Spectroscopic Measurements}

The CD spectra were recorded at room temperature $\left(25^{\circ} \mathrm{C}\right)$ on a Jasco model $\mathrm{J}-500 \mathrm{~A}$ automatic recording circular dichrograph interfaced (IF-500 II) with a IBM AT computer. Cylindrical fused quartz cells of 0.2 , $0.5 \mathrm{~mm}$ pathlengths were used for $\mathrm{CD}$ measurements. The usual instrumental precautions were taken to avoid artefacts. The values are expressed in terms of $[\theta]_{M}$ molar ellipticity ( $\operatorname{deg} \mathrm{cm}^{2} \mathrm{dmol}^{-1}$ ), using the molecular weight of the compounds. 2,2,2-Trifluoroethanol (TFE) was purchased from Fluka (Switzerland); dioxane, methanol and $n$-hexane (spectrophotometric grade) were acquired from Merck (FRG). The peptide concentrations ranged from $2.09 \times 10^{-4}$ to $2.5 \times 10^{-4} \mathrm{M}$.

\section{Bioassays}

Leucinostatin A derivatives showed interesting antimicrobial and antimycotic activities against microorganisms (Table I). Cultures were grown for 48 hours at $30^{\circ} \mathrm{C}$ on nutrient agar broth (one liter contains Constantino ACAS (10 g), $\mathrm{NaCl}(5 \mathrm{~g})$, Constantino peptone $(10 \mathrm{~g}))$ and then centrifuged, washed with physiological solution and adjusted to a final concentration by means of a colorimeter. Cultures were seeded by means multi- point inoculator on $10 \mathrm{~mm}$ Petri dishes containing $19 \mathrm{ml}$ of jelled broth, $1 \mathrm{ml}$ of DMSO and scalar quantities (from 100 to $3 \mu \mathrm{g}$ ) of derivatives per $\mathrm{ml}$ of broth. Control dishes containing only broth-DMSO and broth containing cephaloridine or nistatine were prepared as well and were incubated for 48 hours at $30^{\circ} \mathrm{C}$.

\section{Chemical Modification of Leucinostatin A}

Scheme 1 reports the leucinostatin A homologues obtained by reduction, dehydration and acetylation of the $(4 S, 2 E)$-4-methylhex-2-enoic acid, $(2 S, 4 S, 6 S)$-2amino-6-hydroxy-4-methyl-8-oxodecanoic acid and threo- $\beta$-hydroxy-L-leucine residues.

\section{Leucinostatins A2, A5, A4}

Leucinostatin A hydrochloride, A1 and A $3(70 \mathrm{mg}$ each) were reduced by adding them to a suspension of $5 \% \mathrm{Pd}$ on charcoal in $20 \mathrm{ml}$ of $\mathrm{MeOH}-\mathrm{EtOH}(1: 1)$ previously saturated with $\mathrm{H}_{2}$ at 4 atm at room temperature for 12 hours. Following usual work up, an oily residue was obtained which, after purification by Silica gel column chromatography (eluent $\mathrm{CHCl}_{3}$ $\mathrm{MeOH}$ - conc. $\left.\mathrm{NH}_{4} \mathrm{OH}, 93.1: 6: 0.9\right)$ and several crystallizations from $\mathrm{CH}_{3} \mathrm{CN}$, carefully acidified by gaseous $\mathrm{HCl}$, afforded compounds $\mathrm{A} 2, \mathrm{~A} 5$ and $\mathrm{A} 4$ respectively, $(80 \%$ yield) as hydrochlorides.

Compound A2: white prisms; $m p 173 \sim 175^{\circ} \mathrm{C} ; \operatorname{Rf} 0.78$ $\left(\mathrm{CHCl}_{3}-\mathrm{MeOH}\right.$ - conc. $\left.\mathrm{NH}_{4} \mathrm{OH}, 80: 18: 2\right) ; \mathrm{UV} \lambda_{\text {max }}$ (EtOH): 213 ( $\varepsilon$ 9,325); IR $v_{\max }\left(\mathrm{CDCl}_{3}\right): 3307,1705$ and

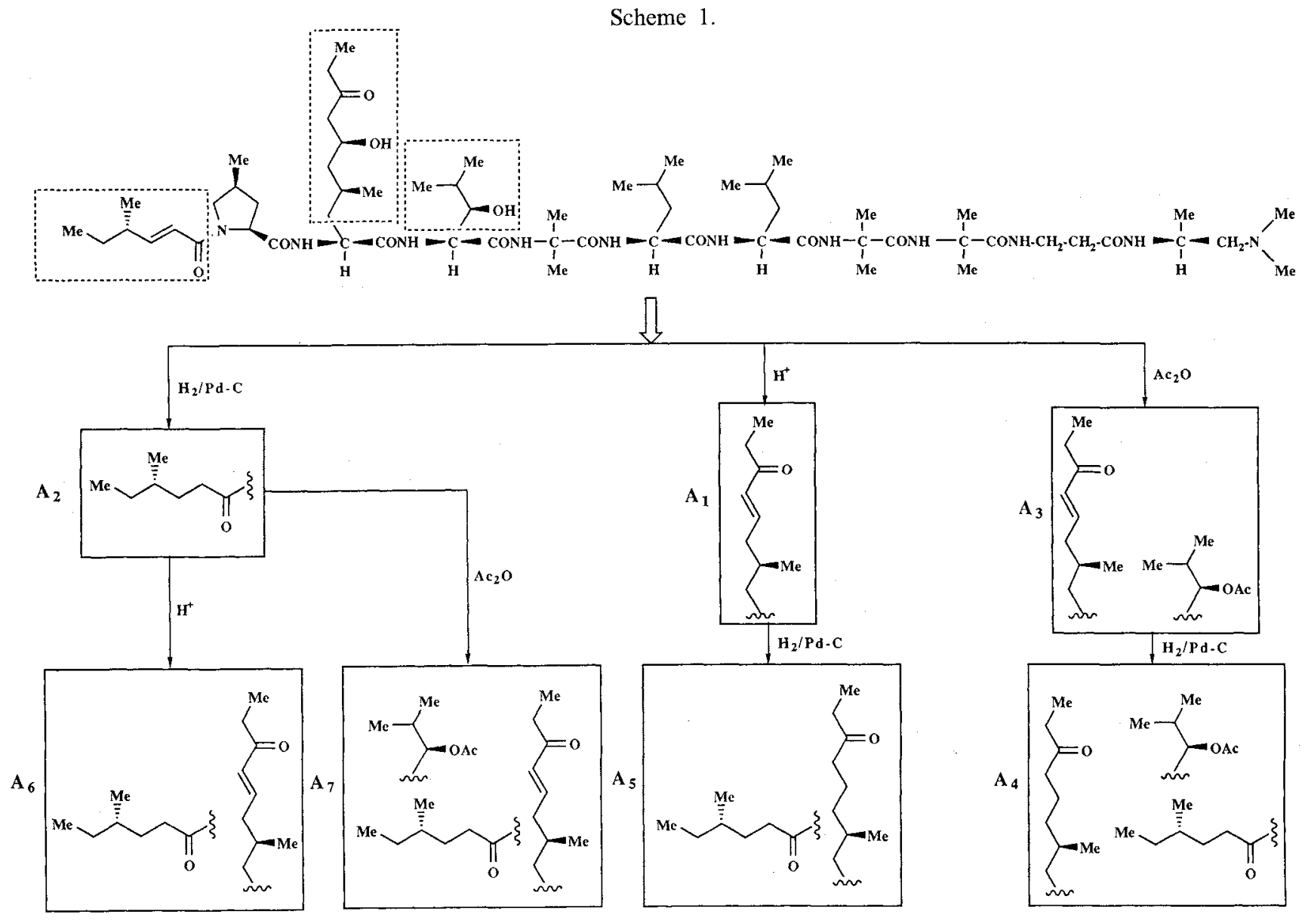


$1654 \mathrm{~cm}^{-1} ;{ }^{1} \mathrm{H}$ NMR $\delta\left(\mathrm{CDCl}_{3}\right): 9.05(1 \mathrm{H}, \mathrm{bs}), 8.32(1 \mathrm{H}$, $\mathrm{d}, J=4 \mathrm{~Hz}), 8.22(1 \mathrm{H}, \mathrm{dd}, J=8$ and $2 \mathrm{~Hz}), 8.1(1 \mathrm{H}, \mathrm{s})$, $7.9(1 \mathrm{H}, \mathrm{s}), 7.8(1 \mathrm{H}, \mathrm{d}, J=4 \mathrm{~Hz}), 7.68(1 \mathrm{H}, \mathrm{s}), 7.40$ $(1 \mathrm{H}, \mathrm{d}, J=6 \mathrm{~Hz}), 7.31(1 \mathrm{H}, \mathrm{d}, J=4 \mathrm{~Hz}), 3.08(3 \mathrm{H}, \mathrm{d}$, $J=2.5 \mathrm{~Hz}), 3.18(3 \mathrm{H}, \mathrm{d}, J=2.5 \mathrm{~Hz}), 1.50(3 \mathrm{H}, \mathrm{s}), 1.48$ $(3 \mathrm{H}, \mathrm{s}), 1.43(3 \mathrm{H}, \mathrm{s}), 1.40(3 \mathrm{H}, \mathrm{s}), 1.38(3 \mathrm{H}, \mathrm{s}), 1.37(3 \mathrm{H}$, s), $1.28(3 \mathrm{H}, \mathrm{d}, J=6.50 \mathrm{~Hz}), 1.12(3 \mathrm{H}, \mathrm{d}, J=6.54 \mathrm{~Hz})$, $1.05(3 \mathrm{H}, \mathrm{d}, J=6.50 \mathrm{~Hz}), 1.03(3 \mathrm{H}, \mathrm{d}, J=7 \mathrm{~Hz}), 1.02(3 \mathrm{H}$, $\mathrm{t}, J=7 \mathrm{~Hz}), 0.96(3 \mathrm{H}, \mathrm{d}, J=7 \mathrm{~Hz}), 0.94(3 \mathrm{H}, \mathrm{d}, J=6 \mathrm{~Hz})$, $0.93(3 \mathrm{H}, \mathrm{d}, J=6 \mathrm{~Hz}), 0.91(3 \mathrm{H}, \mathrm{d}, J=6 \mathrm{~Hz}), 0.90(3 \mathrm{H}$, $\mathrm{t}, J=6.5 \mathrm{~Hz}), 0.88(3 \mathrm{H}, \mathrm{d}, J=6 \mathrm{~Hz}), 0.86(3 \mathrm{H}, \mathrm{d}$, $J=6 \mathrm{~Hz}) ;{ }^{13} \mathrm{C}$ NMR $\delta\left(\mathrm{CDCl}_{3}\right): 211.53(\mathrm{~s}), 177.69(\mathrm{~s})$, $176.68(\mathrm{~s}), 176.48(\mathrm{~s}), 176.06(\mathrm{~s}), 175.83$ (s), 174.76 (s), 174.62 (s), 174.53 (s), 174.34 (s), 172.93 (s), 74.92 (d), $63.96(\mathrm{~d}), 63.60$ (d), $50.80(\mathrm{t}), 45.49(\mathrm{t}), 45.20(2 \times \mathrm{q})$, $31.25(\mathrm{t}), 29.55(\mathrm{t})$.

Compound A5: small white cubes; mp $123 \sim 125^{\circ} \mathrm{C}$; Rf $0.72\left(\mathrm{CHCl}_{3}-\mathrm{MeOH}\right.$ - conc. $\left.\mathrm{NH}_{4} \mathrm{OH}, 80: 18: 2\right)$; $\mathrm{UV} \lambda_{\max }(\mathrm{EtOH}): 213 \mathrm{~nm}(\varepsilon 11,300)$; IR $v_{\max }\left(\mathrm{CHCl}_{3}\right)$ : $3307,1705,1654 \mathrm{~cm}^{-1} ;{ }^{1} \mathrm{H}$ NMR $\delta\left(\mathrm{CDCl}_{3}\right): 9.00(1 \mathrm{H}$, bs), $8.68(1 \mathrm{H}, \mathrm{d}, J=4 \mathrm{~Hz}), 8.48(1 \mathrm{H}, \mathrm{s}), 8.45(1 \mathrm{H}, \mathrm{dd}$, partially obscured), $8.28(1 \mathrm{H}, \mathrm{s}), 8.09(1 \mathrm{H}, \mathrm{d}, J=4 \mathrm{~Hz})$, $7.73(1 \mathrm{H}, \mathrm{s}), 7.58(1 \mathrm{H}, \mathrm{d}, J=6 \mathrm{~Hz}), 7.20(1 \mathrm{H}, \mathrm{d}, J=4 \mathrm{~Hz})$, $3.15(3 \mathrm{H}, \mathrm{d}, J=2.5 \mathrm{~Hz}), 3.05(3 \mathrm{H}, \mathrm{d}, J=2.5 \mathrm{~Hz}), 1.63$ $(3 \mathrm{H}, \mathrm{s}), 1.62(3 \mathrm{H}, \mathrm{s}), 1.58(3 \mathrm{H}, \mathrm{s}), 1.50(3 \mathrm{H}, \mathrm{s}), 1.49(3 \mathrm{H}$, s), $1.48(3 \mathrm{H}, \mathrm{s}), 1.30(3 \mathrm{H}, \mathrm{d}, J=6.5 \mathrm{~Hz}), 1.13(3 \mathrm{H}, \mathrm{d}$, $J=6.50 \mathrm{~Hz}), 1.05(3 \mathrm{H}, \mathrm{d}, J=6.5 \mathrm{~Hz}), 1.02(3 \mathrm{H}, \mathrm{t}$, $J=7.00 \mathrm{~Hz}), 0.98(3 \mathrm{H}, \mathrm{d}, J=7.0 \mathrm{~Hz}), 0.97(3 \mathrm{H}, \mathrm{d}$, $J=6.0 \mathrm{~Hz}), 0.90 \sim 0.80(18 \mathrm{H}, \mathrm{m}) ;{ }^{13} \mathrm{C} \mathrm{NMR} \delta\left(\mathrm{CDCl}_{3}\right)$ : $212.64(\mathrm{~s}), 176.62$ (s), 176.11 (s), 175.14 (s), 174.98 (s), 174.69 (s), 174.46 (s), 173.54 (s), 172.74 (s), 172.31 (s), $75.60(\mathrm{~d}), 63.60(\mathrm{~d}), 45.41(2 \times \mathrm{q}), 42.46(\mathrm{t}), 36.88(2 \times \mathrm{t})$, $31.38(\mathrm{t}), 29.64(\mathrm{t})$.

Compound A4: white amorphous powder; mp $100 \sim 102^{\circ} \mathrm{C}$; Rf $0.72\left(\mathrm{CHCl}_{3}-\mathrm{MeOH}\right.$ - conc. $\mathrm{NH}_{4} \mathrm{OH}$, $80: 18: 2)$; UV $\lambda_{\max }(\mathrm{EtOH}): 213 \mathrm{~nm}(\varepsilon 10,628)$; IR $v_{\max }$ $\left(\mathrm{CHCl}_{3}\right): 3317,1705$ and $1645 \mathrm{~cm}^{-1} ;{ }^{1} \mathrm{H} \mathrm{NMR} \delta$ $\left(\mathrm{CDCl}_{3}\right): 8.85(1 \mathrm{H}, \mathrm{m}), 8.30(1 \mathrm{H}, \mathrm{s}), 8.22(1 \mathrm{H}, \mathrm{dd}, J=8$ and $2 \mathrm{~Hz}), 7.90(1 \mathrm{H}, \mathrm{s}), 7.85(1 \mathrm{H}, \mathrm{d}, J=4 \mathrm{~Hz}), 7.70(1 \mathrm{H}$, s), $7.65(1 \mathrm{H}, \mathrm{d}, J=4 \mathrm{~Hz}), 7.58(1 \mathrm{H}, \mathrm{d}, J=4 \mathrm{~Hz}), 7.39(1 \mathrm{H}$, $\mathrm{d}, J=6 \mathrm{~Hz}), 5.10(1 \mathrm{H}, \mathrm{dd}, J=4.5$ and $7.5 \mathrm{~Hz}), 3.15$ $(2 \times 3 \mathrm{H}, \mathrm{s}), 2.08(3 \mathrm{H}, \mathrm{s}), 1.5(3 \mathrm{H}, \mathrm{s}), 1.49(3 \mathrm{H}, \mathrm{s}), 1.47$ $(3 \mathrm{H}, \mathrm{s}), 1.43(6 \mathrm{H}, \mathrm{s}), 1.40(3 \mathrm{H}, \mathrm{s}), 1.23(3 \mathrm{H}, \mathrm{d}, J=6.5 \mathrm{~Hz})$, $1.13(3 \mathrm{H}, \mathrm{d}, J=6.55 \mathrm{~Hz}), 1.05(3 \mathrm{H}, \mathrm{d}, J=6.5 \mathrm{~Hz}), 1.01$ $(3 \mathrm{H}, \mathrm{t}, J=7 \mathrm{~Hz}), 0.98 \sim 0.8(24 \mathrm{H}, \mathrm{m}) ;{ }^{13} \mathrm{C}$ NMR $\delta$ $\left(\mathrm{CDCl}_{3}\right): 212.02(\mathrm{~s}), 177.65(\mathrm{~s}), 176.31$ (s), 176.17 (s), 176.09 (s), 175.75 (s), 174.50 (s), 174.45 (s), 174.28 (s), 174.17 (s), 171.60 (s), 170.78 (s), 75.61 (d), 63.30 (d), $44.41(2 \times \mathrm{q}), 42.58(\mathrm{t}), 37.40(\mathrm{t}), 36.36(\mathrm{t}), 31.84(\mathrm{t}), 29.55$ (t), $19.02(\mathrm{q})$.

\section{Leucinostatins A1, A6}

Leucinostatin A hydrochloride and A2 (100 mg each) in $\mathrm{CHCl}_{3}(30 \mathrm{ml})$ were treated with gaseous $\mathrm{HCl}$ for 10 minutes and then deluted with $\mathrm{CHCl}_{3}(60 \mathrm{ml})$. The solutions brought to dryness at reduced pressure left oily yellow residues which were purified by column chromatography (eluent $\mathrm{CHCl}_{3}-\mathrm{MeOH}$-conc. $\mathrm{NH}_{4} \mathrm{OH}$,
93.1:6:0.9) and eventually crystallized by $\mathrm{CH}_{3} \mathrm{CN}$, carefully acidified by gaseous $\mathrm{HCl}$, to afford compounds A1 and A6 respectively ( $85 \%$ yield) as hydrochloride salts. Compound A6 was further purified by flash column chromatography using the usual ternary eluent.

Compound A1: white small cubes; mp $145 \sim 147^{\circ} \mathrm{C}$; Rf $0.77\left(\mathrm{CHCl}_{3}-\mathrm{MeOH}\right.$ - conc. $\left.\mathrm{NH}_{4} \mathrm{OH}, 80: 18: 2\right) ; \mathrm{UV}$ $\lambda_{\text {max }}$ (EtOH): $219.5 \mathrm{~nm}(\varepsilon 29,629)$ and $211 \mathrm{~nm}(\varepsilon$ 15,946); IR $v_{\max }\left(\mathrm{CHCl}_{3}\right): 3307$ and $1654 \mathrm{~cm}^{-1} ;{ }^{1} \mathrm{H}$ NMR $\delta$ $\left(\mathrm{CDCl}_{3}\right): 9.12(1 \mathrm{H}, \mathrm{bs}), 8.68(1 \mathrm{H}, \mathrm{d}, J=4 \mathrm{~Hz}), 8.29(1 \mathrm{H}$, dd, partially obscured), $8.28(1 \mathrm{H}, \mathrm{s}), 8.02(1 \mathrm{H}, \mathrm{s}), 7.95$ $(1 \mathrm{H}, \mathrm{d}, J=4 \mathrm{~Hz}), 7.68(1 \mathrm{H}, \mathrm{s}), 7.42(1 \mathrm{H}, \mathrm{d}, J=6 \mathrm{~Hz})$, $7.22(1 \mathrm{H}, \mathrm{d}, J=4 \mathrm{~Hz}), 6.88(1 \mathrm{H}, \mathrm{m}), 6.73(1 \mathrm{H}, \mathrm{dd}, J=15.4$ and $7.4 \mathrm{~Hz}), 6.20(1 \mathrm{H}, \mathrm{d}, J=15.4 \mathrm{~Hz}), 6.05(1 \mathrm{H}, \mathrm{d}$, $J=15.4 \mathrm{~Hz}), 3.15(3 \mathrm{H}, \mathrm{d}, J=2.5 \mathrm{~Hz}), 3.07(3 \mathrm{H}, \mathrm{d}, J=$ $2.5 \mathrm{~Hz}), 1.58(3 \mathrm{H}, \mathrm{s}), 1.57(3 \mathrm{H}, \mathrm{s}), 1.49(3 \mathrm{H}, \mathrm{s}), 1.47(3 \mathrm{H}$, s), $1.44(3 \mathrm{H}, \mathrm{s}), 1.43(3 \mathrm{H}, \mathrm{s}), 1.23(3 \mathrm{H}, \mathrm{d}, J=6.5 \mathrm{~Hz})$, $1.13(3 \mathrm{H}, \mathrm{d}, J=6.55 \mathrm{~Hz}), 1.07(3 \mathrm{H}, \mathrm{d}, J=6.50 \mathrm{~Hz}), 1.05$ $(3 \mathrm{H}, \mathrm{d}, J=7 \mathrm{~Hz}), 1.03(3 \mathrm{H}, \mathrm{t}, J=7 \mathrm{~Hz}), 0.98(3 \mathrm{H}, \mathrm{d}$, $J=7 \mathrm{~Hz}), 0.96(3 \mathrm{H}, \mathrm{d}, J=6 \mathrm{~Hz}), 0.94(3 \mathrm{H}, \mathrm{d}, J=6 \mathrm{~Hz})$, $0.92(3 \mathrm{H}, \mathrm{d}, J=6 \mathrm{~Hz}), 0.91(3 \mathrm{H}, \mathrm{t}, J=6.5 \mathrm{~Hz}), 0.90(3 \mathrm{H}$, $\mathrm{d}, J=6 \mathrm{~Hz}), 0.88(3 \mathrm{H}, \mathrm{d}, J=6 \mathrm{~Hz}) ;{ }^{13} \mathrm{C} \mathrm{NMR} \delta\left(\mathrm{CDCl}_{3}\right)$ : 201.02 (s), 177.71 (s), 176.54 (s), 175.95 (s), 175.81 (s), 175.75 (s), 174.45 (s), 174.34 (s), 174.23 (s), 172.64 (s), 167.03 (s), 151.23 (d), 145.18 (d), 131.61 (d), 120.31 (d), $74.34(d), 63.60(d), 46.1(2 \times q), 36.80(t)$.

Compound A6: amorphous white powder; $\mathrm{mp}$ $110 \sim 112^{\circ} \mathrm{C}$; Rf $0.77\left(\mathrm{CHCl}_{3}-\mathrm{MeOH}\right.$ - conc. $\mathrm{NH}_{4} \mathrm{OH}$, 80:18:2); UV $\lambda_{\text {max }}$ (EtOH): $214.7 \mathrm{~nm}(\varepsilon$ 14,700) and $210.5 \mathrm{~nm}(\varepsilon 4,732) ; \mathrm{IR} v_{\max }\left(\mathrm{CHCl}_{3}\right): 3309$ and $1654 \mathrm{~cm}^{-1}$; ${ }^{1} \mathrm{H}$ NMR $\delta\left(\mathrm{CDCl}_{3}\right): 8.77(1 \mathrm{H}, \mathrm{bs}), 8.58(1 \mathrm{H}, \mathrm{d}, J=$ $4 \mathrm{~Hz}), 8.12(1 \mathrm{H}, \mathrm{s}), 8.10(1 \mathrm{H}, \mathrm{dd}$, partially obscured), $7.95(1 \mathrm{H}, \mathrm{s}), 7.90(1 \mathrm{H}, \mathrm{d}, J=4 \mathrm{~Hz}), 7.50(1 \mathrm{H}, \mathrm{s}), 7.30(1 \mathrm{H}$, $\mathrm{d}, J=6 \mathrm{~Hz}), 7.22(1 \mathrm{H}, \mathrm{d}, J=4 \mathrm{~Hz}), 6.85(1 \mathrm{H}, \mathrm{m}), 6.07$ $(1 \mathrm{H}, \mathrm{d}, J=15.4 \mathrm{~Hz}), 2.9(2 \times 3 \mathrm{H}, \mathrm{s}), 1.54(3 \mathrm{H}, \mathrm{s}), 1.53$ $(3 \mathrm{H}, \mathrm{s}), 1.50(3 \mathrm{H}, \mathrm{s}), 1.48(3 \mathrm{H}, \mathrm{s}), 1.47(3 \mathrm{H}, \mathrm{s}), 1.43(3 \mathrm{H}$, s), $1.23(3 \mathrm{H}, \mathrm{d}, J=6.5 \mathrm{~Hz}), 1.13(3 \mathrm{H}, \mathrm{d}, J=6.5 \mathrm{~Hz}), 1.13$ $(3 \mathrm{H}, \mathrm{d}, J=7.0 \mathrm{~Hz}), 1.08(3 \mathrm{H}, \mathrm{d}, J=6.50 \mathrm{~Hz}), 1.06(3 \mathrm{H}, \mathrm{d}$, $J=6 \mathrm{~Hz}), 1.05(3 \mathrm{H}, \mathrm{d}, J=6 \mathrm{~Hz}), 0.96(3 \mathrm{H}, \mathrm{d}, J=6 \mathrm{~Hz})$, $0.95(3 \mathrm{H}, \mathrm{d}, J=6 \mathrm{~Hz}), 0.93(3 \mathrm{H}, \mathrm{t}, J=6.5 \mathrm{~Hz}), 0.92(3 \mathrm{H}$, $\mathrm{d}, J=6 \mathrm{~Hz}), 0.90(3 \mathrm{H}, \mathrm{d}, J=6 \mathrm{~Hz}), 0.88(3 \mathrm{H}, \mathrm{d}, J=6 \mathrm{~Hz})$; ${ }^{13} \mathrm{C} \mathrm{NMR} \delta\left(\mathrm{CDCl}_{3}\right): 201.2(\mathrm{~s}), 177.36(\mathrm{~s}), 176.62(\mathrm{~s})$, 175.86 (s), 175.78 (s), 175.72 (s), 174.65 (s), 174.59 (s), 174.40 (s), 173.89 (s), 172.76 (s), 145.31 (d), 131.69 (d), $74.47(\mathrm{~d}), 63.60(\mathrm{~d}), 44.85(2 \times \mathrm{q}), 36.9(\mathrm{t}), 31.36(\mathrm{t}), 29.44$ (t).

\section{Leucinostatins A3, A7}

Leucinostatin A hydrochloride and A2 (100 $\mathrm{ml}$ each), dissolved with dry pyridine $(2 \mathrm{ml})$ and acetic anhydride $(1 \mathrm{ml})$ were stirred for 24 hours at room temperature and then poured in iced water. The cloudy solutions were repeatedly extracted with $\mathrm{CHCl}_{3}(5 \times 30 \mathrm{ml})$. The combined organic layers, dried over anhydrous $\mathrm{Na}_{2} \mathrm{SO}_{4}$, were brought to dryness at reduced pressure and the oily yellow residues were purified by flash column chromato- 
graphy (eluent $\mathrm{CHCl}_{3}-\mathrm{MeOH}$-conc. $\mathrm{NH}_{4} \mathrm{OH}, 93.1$ : $6: 0.9)$ in a nitrogen atmosphere to afford compounds A3 and A7 respectively ( $90 \%$ yield) as free base forms.

Compound A3: white amorphous powder; $\mathrm{mp}$ $98 \sim 100^{\circ} \mathrm{C}$, Rf $0.74\left(\mathrm{CHCl}_{3}-\mathrm{MeOH}\right.$ - conc. $\mathrm{NH}_{4} \mathrm{OH}$, $80: 18: 2)$, UV $\lambda_{\text {max }}(E t O H): 219 \mathrm{~nm}(\varepsilon 26,420)$ and $211 \mathrm{~nm}$ $(\varepsilon 16,060)$; IR $v_{\max }\left(\mathrm{CHCl}_{3}\right): 3321,1735$ and $1634 \mathrm{~cm}^{-1}$; ${ }^{1} \mathrm{H}$ NMR $\delta\left(\mathrm{CDCl}_{3}\right): 8.48(1 \mathrm{H}, \mathrm{bs}), 8.10(1 \mathrm{H}, \mathrm{s}), 7.82$ $(2 \mathrm{H}, \mathrm{m}), 7.68(1 \mathrm{H}, \mathrm{s}), 7.49(2 \mathrm{H}, \mathrm{m}), 7.32(1 \mathrm{H}, \mathrm{s}), 7.10$ $(1 \mathrm{H}, \mathrm{d}, J=6 \mathrm{~Hz}), 6.88(1 \mathrm{H}, \mathrm{m}), 6.73(1 \mathrm{H}, \mathrm{dd}, J=15.4$ and $7.4 \mathrm{~Hz}), 6.15(1 \mathrm{H}, \mathrm{d}, J=15.4 \mathrm{~Hz}), 6.05(1 \mathrm{H}, \mathrm{d}$, $J=15.4 \mathrm{~Hz}), 5.10(1 \mathrm{H}, \mathrm{dd}, J=4.5$ and $7.5 \mathrm{~Hz}), 2.65(6 \mathrm{H}$, s), $2.06(3 \mathrm{H}, \mathrm{s}), 1.49(3 \mathrm{H}, \mathrm{s}), 1.48(3 \mathrm{H}, \mathrm{s}), 1.46(6 \mathrm{H}, \mathrm{s})$, $1.44(3 \mathrm{H}, \mathrm{s}), 1.42(3 \mathrm{H}, \mathrm{s}), 1.23(3 \mathrm{H}, \mathrm{d}, J=6.5 \mathrm{~Hz}), 1.13$ $(3 \mathrm{H}, \mathrm{d}, J=6.55 \mathrm{~Hz}), 1.05(3 \mathrm{H}, \mathrm{d}, J=6.5 \mathrm{~Hz}), 1.02(3 \mathrm{H}$, $\mathrm{t}, J=7 \mathrm{~Hz}), 1.00(3 \mathrm{H}, \mathrm{d}, J=6.0 \mathrm{~Hz}), 0.92 \sim 0.81(21 \mathrm{H}$, $\mathrm{m}) ;{ }^{13} \mathrm{C}$ NMR $\delta\left(\mathrm{CDCl}_{3}\right): 200.95(\mathrm{~s}), 176.56(\mathrm{~s}), 176.06$ (s), $175.10(\mathrm{~s}), 175.02(2 \mathrm{~s}), 174.39(\mathrm{~s}), 174.00(\mathrm{~s}), 172.90$ (s), $171.06(\mathrm{~s}), 170.68(\mathrm{~s}), 166.75(\mathrm{~s}), 152.72$ (d), 144.76 (d), 131.55 (d), 119.71 (d), 75.98 (d), 63.15 (d), 44.99 $(2 \times \mathrm{q}), 39.8(\mathrm{t}), 20.7(\mathrm{q})$.

Compound A7: white amorphous powder; $\mathrm{mp}$ $106 \sim 110^{\circ} \mathrm{C}$; Rf $0.75\left(\mathrm{CHCl}_{3}-\mathrm{MeOH}\right.$ - conc. $\mathrm{NH}_{4} \mathrm{OH}$, $80: 18: 2)$; UV $\lambda_{\max }$ (EtOH): $214.6 \mathrm{~nm}(\varepsilon 15,810) ; \mathrm{IR} v_{\max }$ $\left(\mathrm{CHCl}_{3}\right): 3318,1735$ and $1654 \mathrm{~cm}^{-1} ;{ }^{1} \mathrm{H}$ NMR $\left(\mathrm{CDCl}_{3}\right)$ : $9.55(1 \mathrm{H}, \mathrm{bs}), 8.35(1 \mathrm{H}, \mathrm{dd}$, partially obscured $), 8.33(1 \mathrm{H}$, s), $8.01(1 \mathrm{H}, \mathrm{d}, J=4 \mathrm{~Hz}), 7.80(1 \mathrm{H}, \mathrm{s}), 7.77(1 \mathrm{H}, \mathrm{s}), 7.70$ $(1 \mathrm{H}, \mathrm{d}, J=4 \mathrm{~Hz}), 7.64(1 \mathrm{H}, \mathrm{d}, J=4 \mathrm{~Hz}), 7.49(1 \mathrm{H}, \mathrm{d}$, $J=6 \mathrm{~Hz}), 6.90(1 \mathrm{H}, \mathrm{m}), 6.05(1 \mathrm{H}, \mathrm{d}, J=15.4 \mathrm{~Hz}), 5.12$ $(1 \mathrm{H}, \mathrm{dd}, J=4.5$ and $7.5 \mathrm{~Hz}), 2.33(6 \mathrm{H}, \mathrm{s}), 2.18(3 \mathrm{H}, \mathrm{s})$,
$1.52(3 \mathrm{H}, \mathrm{s}), 1.50(6 \mathrm{H}, \mathrm{s}), 1.47(6 \mathrm{H}, \mathrm{s}), 1.40(3 \mathrm{H}, \mathrm{s}), 1.28$ $(3 \mathrm{H}, \mathrm{d}, J=6.5 \mathrm{~Hz}), 1.18(3 \mathrm{H}, \mathrm{d}, J=6.55 \mathrm{~Hz}), 1.08(3 \mathrm{H}$, d, $J=6.5 \mathrm{~Hz}), 1.02(3 \mathrm{H}, \mathrm{t}, J=7.0 \mathrm{~Hz}), 1.00 \sim 0.87(24 \mathrm{H}$, $\mathrm{m}) ;{ }^{13} \mathrm{C} \mathrm{NMR} \delta\left(\mathrm{CDCl}_{3}\right): 200.80(\mathrm{~s}), 176.089(\mathrm{~s}), 176.0$ (s), $174.76(2 \times \mathrm{s}), 174.39(\mathrm{~s}), 174.14(\mathrm{~s}), 173.94(\mathrm{~s}), 172.33$ (s), $170.95(\mathrm{~s}), 170.86(2 \times \mathrm{s}), 144.47$ (d), 131.60 (d), 76.06 (d), $45.05(2 \times q), 36.90(t), 31.39(t), 29.55(t), 20.76(q)$.

\section{Results and Discussion}

Fig. 1 represents the CD spectra of leucinostatin A hydrochloride homologues $\mathrm{A} 1, \mathrm{~A} 2$ and $\mathrm{A} 5$ in solvents of different polarity. They show the same spectral behaviour observed for leucinostatin A hydrochloride ${ }^{8)}$. In TFE the CD profile (negative maximum at $203 \mathrm{~nm}$ and a large shoulder at $210 \sim 220 \mathrm{~nm}$ ) indicates the existence of a conformational equilibrium between aperiodic structure and folded conformations $\left(3_{10}\right.$ or $\alpha$-helical structures). This equilibrium shifts towards populations in ordered conformation in the less polar solvent $\mathrm{MeOH}$ and, chiefly, in the more apolar dioxane$n$-hexane $(1: 1, \mathrm{v} / \mathrm{v})$ mixture. In this solvent the $\mathrm{CD}$ band of the amide $\pi-\pi^{*}$ transition moved to $208 \mathrm{~nm}$ and the negative maximum of the amide $n-\pi^{*}$ transition is well resolved at $220 \sim 222 \mathrm{~nm}$ ( $\alpha$-helix structure). This spectral behaviour means that chemical modifications do not influence macroscopically the ordered conformation

Fig. 1. CD spectra of homologues A1, A2, A5 in TFE (curve 1), MeOH (curve 2) and dioxane- $n$-hexane $(1: 1$, v/v) (curve 3).
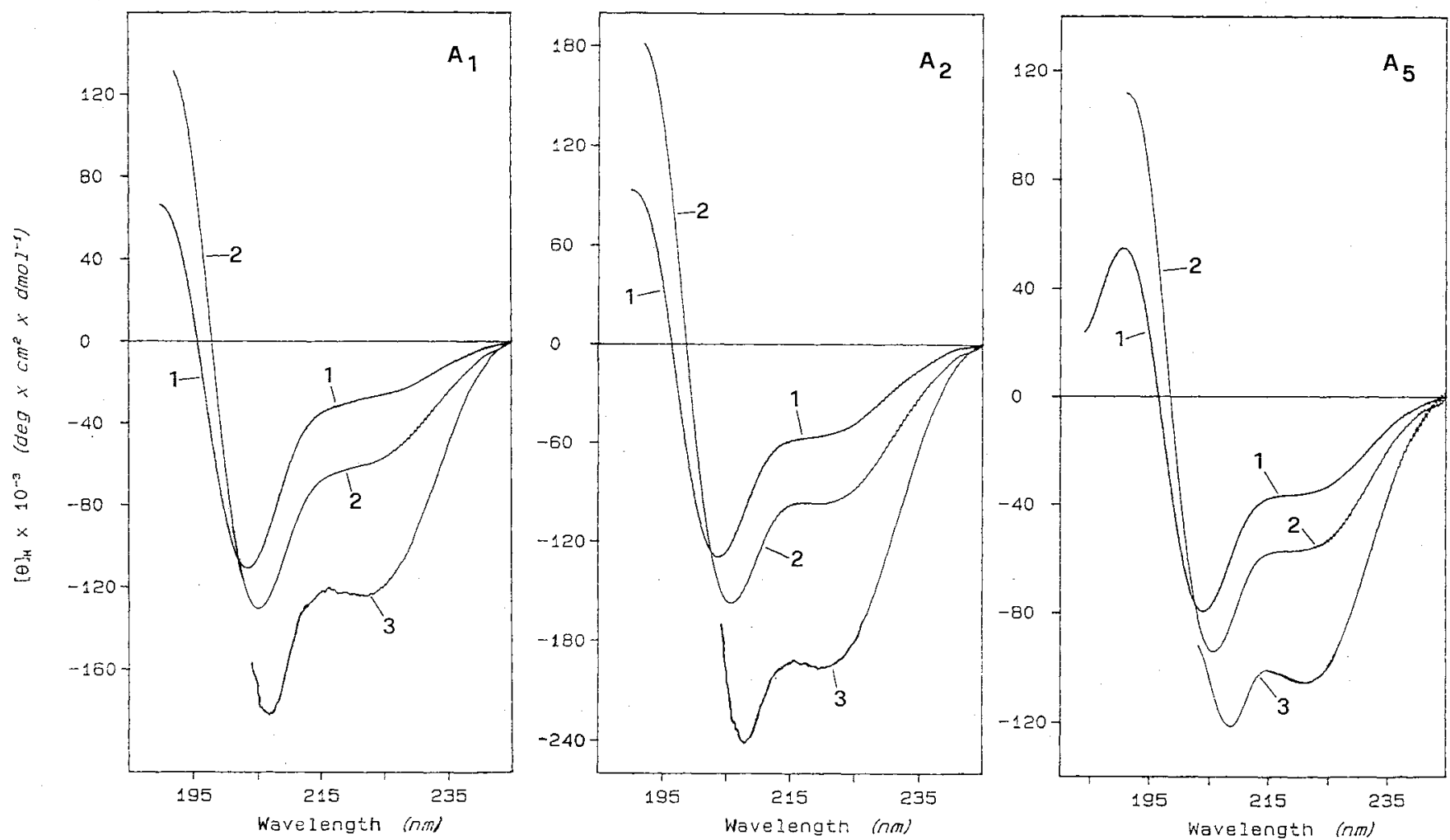
Table 1. Antibacterial and antimycotic activity of leucinostatin $\mathrm{A} \cdot \mathrm{HCl}$ homologues. $(\mathrm{MIC}, \mu \mathrm{g} / \mathrm{ml})$

\begin{tabular}{|c|c|c|c|c|c|c|c|c|}
\hline \multirow{2}{*}{ Microorganism } & \multicolumn{8}{|c|}{ Compounds } \\
\hline & $\mathrm{A}^{*}$ & Al & $\mathrm{A} 2$ & A3 & A4 & A5 & A6 & A7 \\
\hline Bacillus subtilis ICI & 3 & $<3$ & $<3$ & $<3$ & 3 & 3 & 12 & 6 \\
\hline Micrococcus luteus 9341 & $<3$ & 3 & $<3$ & $<3$ & $<3$ & $<3$ & $<3$ & $<3$ \\
\hline Bacillus subtilis var. niger & 3 & 3 & 12 & $<3$ & $<3$ & 3 & 12 & $<3$ \\
\hline Bacillus cereus B43 1335 & 12 & 12 & 12 & $<3$ & 6 & 6 & 12 & 6 \\
\hline Staphilococcus aureus & 12 & 12 & 12 & 6 & 6 & 3 & 12 & 6 \\
\hline Pseudomonas aeruginosa 6750 & $>100$ & $>100$ & $>100$ & $>100$ & $>100$ & $>100$ & $>100$ & $>100$ \\
\hline Salmonella typhimurium & $>100$ & $>100$ & $>100$ & $>100$ & $>100$ & $>100$ & $>100$ & $>100$ \\
\hline Proteus vulgaris & $>100$ & $>100$ & $>100$ & $>100$ & $>100$ & $>100$ & $>100$ & $>100$ \\
\hline Escherichia coli 982 & $>100$ & $>100$ & $>100$ & $>100$ & $>100$ & $>100$ & $>100$ & $>100$ \\
\hline Citrobacter freundii & $>100$ & $>100$ & $>100$ & $>100$ & $>100$ & $>100$ & $>100$ & $>100$ \\
\hline Pseudomonas fluorescens $\mathrm{C} 3$ & $>100$ & $>100$ & $>100$ & $>100$ & $>100$ & $>100$ & $>100$ & $>100$ \\
\hline Candida albicans CBS 562 & $<3$ & $<3$ & $<3$ & 50 & 3 & $<3$ & 12 & 12 \\
\hline Candida tropicalis 5711 IMAT & 25 & 25 & 25 & 100 & 25 & 12 & 25 & 25 \\
\hline Candida krusei CBS 1910 & 25 & 50 & 25 & $>100$ & 100 & 25 & 50 & $>100$ \\
\hline Cryptococcus laurentii 4685 & 50 & 100 & 25 & 100 & 100 & 100 & 50 & $>100$ \\
\hline Cryptococcus neoformans 4711 IMAT & $<3$ & $<3$ & $<3$ & 100 & 3 & 3 & 12 & 25 \\
\hline
\end{tabular}

* Leucinostatin $\mathrm{A} \cdot \mathrm{HCl}$.

of the parent natural compound. The spectroscopic results parallel the biological activities (Table 1) which do not show any significative variations if compared to those of leucinostatin A hydrochloride, with the exception of A5 which resulted more active against Staphilococcus aureus. Interestingly CD spectra of A4 (Fig. 2) revealed the presence of two sharp negative bands centered at 208 and $222 \mathrm{~nm}$ in TFE and $\mathrm{MeOH}$ too, whose intensities approach those observed in dioxane$n$-hexane solution. This indicates a remarkable increase of an ordered molecular population in these solvents probably due to the acetylation of the threo- $\beta$-hydroxyL-leucine residue. In fact the lack of a free hydroxy group prevents the formation of an intermolecular hydrogen bond with the solvent and, at the same time, increases the lipophile character of the molecule cooperating thus to the formation of an ordered structure. The CD spectra of compounds A3 and A7 (Fig. 3), insoluble in buffer solution, show in TFE and $\mathrm{MeOH}$ a similar behaviour of A4 while in a very apolar medium, such as dioxane - $n$-hexane the equilibrium ordered conformation versus disordered conformation is clearly displaced towards the latter. Since NMR spectra undoubtedly indicate that these compounds are in the free base and not in the hydrochloride form, it was inferred that molecules with positively charged C-terminal moiety assume an ordered conformation in an apolar medium and vice versa. Such a hypothesis was confirmed by performing CD spectra of leucinostatin A free base (Fig. 4 ); in this case a similar behaviour was observed, in fact by dissolving this compound in dioxane- $n$-hexane, a
Fig. 2. CD spectra of homologues A4 in TFE (curve 1), $\mathrm{MeOH}$ (curve 2) and dioxane - $n$-hexane $(1: 1, \mathrm{v} / \mathrm{v}$ ) (curve 3 ).

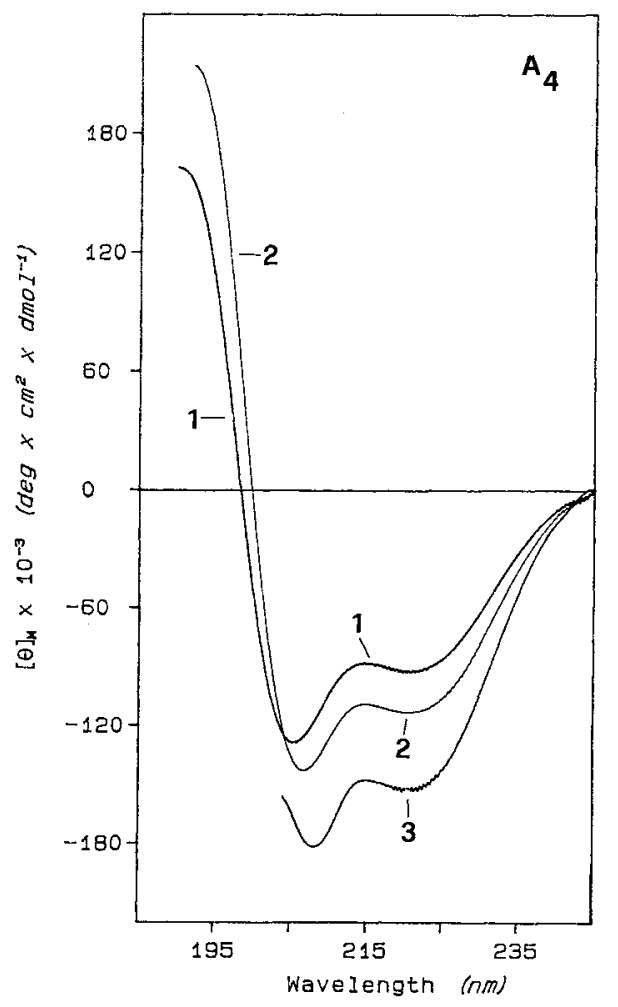

complete inversion of the behaviour, as compared to what showed by leucinostatin A hydrochloride, was noticed. In acqueous buffer medium, the spectrum profile of leucinostatin A free base, as in the case of hydrochloride form ${ }^{8)}$, indicates the presence of a limited molecular population in ordered structure (low intensities of the negative bands and blue shift of $\pi-\pi^{*}$ transition). By comparing these data with the biological activities 
Fig. 3. CD spectra of homologues A3, A7 in TFE (curve 1), $\mathrm{MeOH}$ (curve 2) and dioxane- $n$-hexane $(1: 1, \mathrm{v} / \mathrm{v})$ (curve 3).
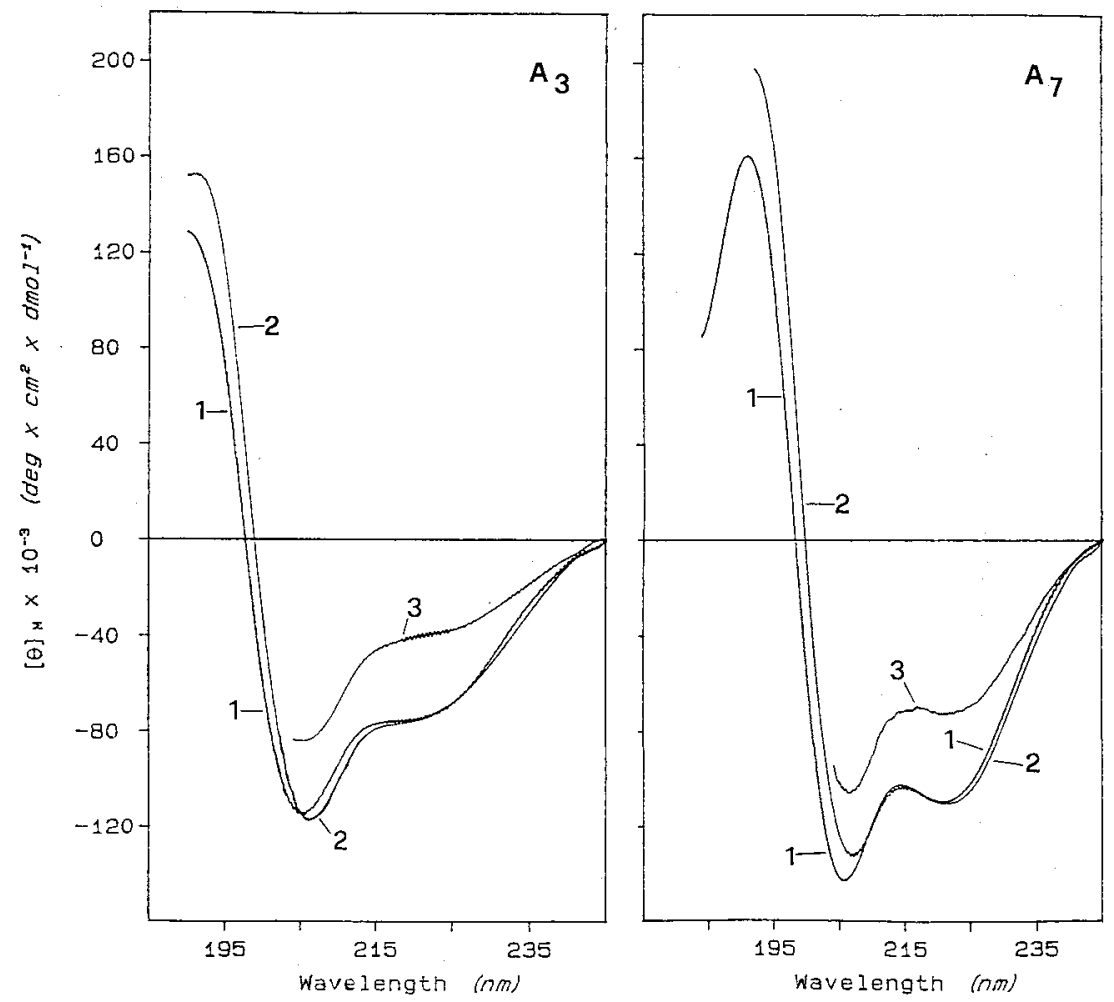

Fig. 4. CD spectra of leucinostatin A free base in TFE (curve 1), $\mathrm{MeOH}$ (curve 2), dioxane - $n$-hexane $(1: 1, \mathrm{v} / \mathrm{v})$ (curve 3 ) and in TFE $2 \mathrm{~mm}$ phosphate buffer, $\mathrm{pH} 7.4(1: 19, \mathrm{v} / \mathrm{v})$ (curve 4).

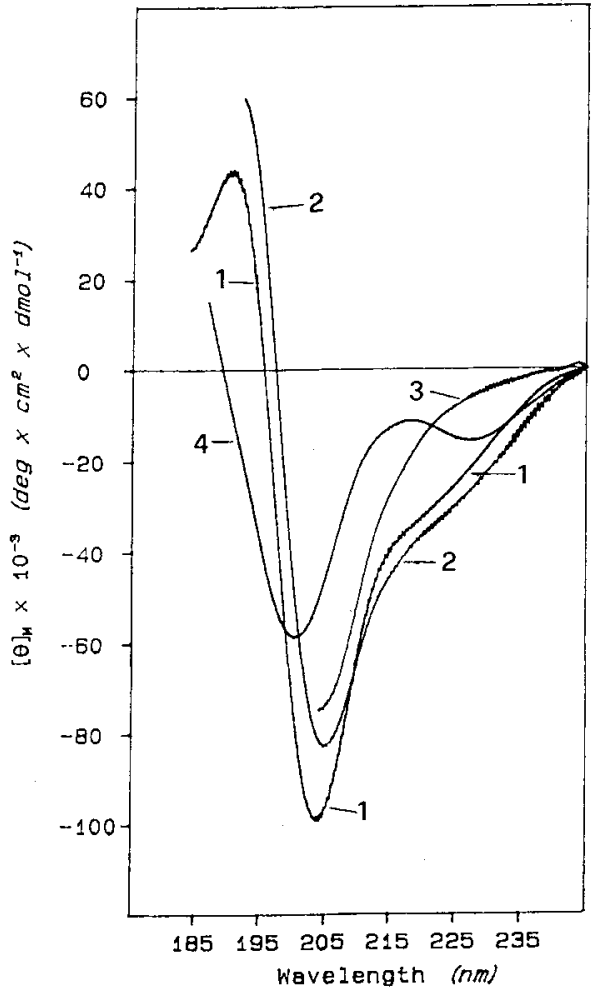

Fig. 5. CD spectra of homologues A6 in TFE (curve 1), $\mathrm{MeOH}$ (curve 2) and dioxane- $n$-hexane $(1: 1, \mathrm{v} / \mathrm{v})$ (curve 3).

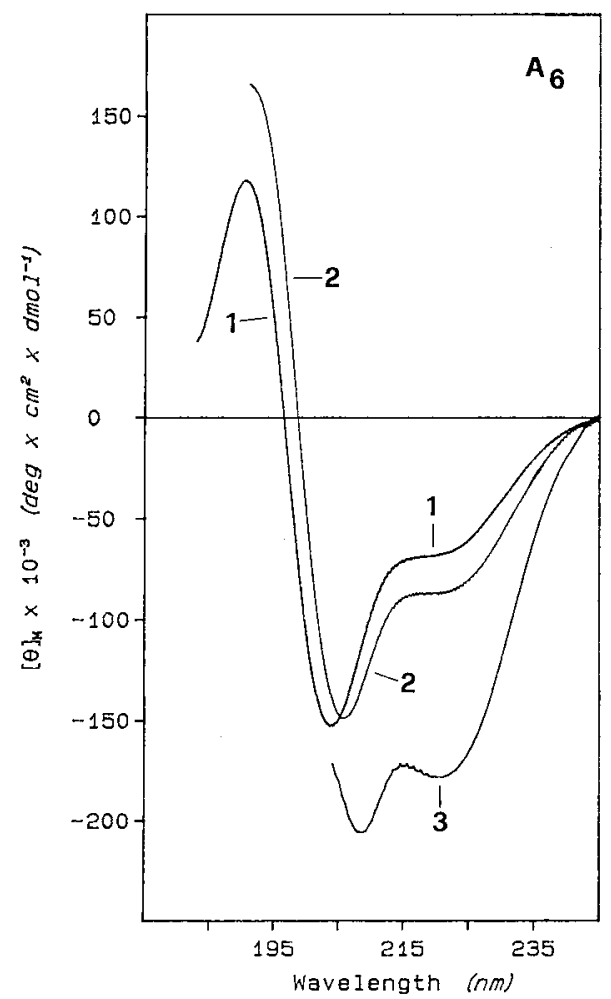


(Table 1) it is possible to argue that in addition to the presence of a charged C-terminal moiety, which appeared at the first glance indispensable for the activity, is also important the higher lipophyle character of these compounds. In fact A3, A7 and A4 show a higher antimicrobial and a lower antifungal activity if compared to what displayed by the parent leucinostatin A hydrochloride. The apparent anomalous behaviour noticed in the CD spectra of compound A6 (Fig. 5) is due to the fact that A6 exists in the hydrochloride-free base form mixture (75.25) as inferred from leucinostatin A NMR shift titration experiments ${ }^{12}$. In fact the CD curves in $\mathrm{MeOH}$ and TFE approach those observed for compounds $\mathrm{A} 3$ and $\mathrm{A} 7$ while the CD curve in dioxane$n$-hexane $(1: 1, \mathrm{v} / \mathrm{v})$ is very similar to that one shown by compounds A1, A2, A4 and A5. Accordingly with these findings, A6 shows a mild activity against Gram-positive bacteria and no significant differences in activity against fungi if compared to that observed for the hydrochloride homologues.

\section{Acknowledgment}

This work has been supported by the grant of the National Research Council (C.N.R.) "Progetto Finalizzato-Chimica Fine e Secondaria II"

\section{References}

1) Fukushima, K.; T. Arai, Y. Mori, M. Tsuboi \& M. SuZUKI: Studies on peptide antibiotics, Leucinostatins II. The structures of Leucinostatins A and B. J. Antibiotics 36: $1613 \sim 1630,1983$

2) Casinovi, C. G.; L. Tuttobello, C. Rossi \& Z. Benciari: Structural elucidation of the phytotoxic antibitic peptides produced by submerged cultures of Paecilomyces marquandii (Massee) Hughes. Phytopathol. Mediterr. 22: $103 \sim 106,1983$

3) Isogar, A.; A. Suzuki, S. Tamura, S. Higashikawa \&
S. KuYAma: Structure of a peptidal antibiotic P168 produced by Paecilomyces lilacinus (Thom) Samson. J. Chem. Soc. Perkin Trans. I: $1405 \sim 1411,1984$

4) Casinovi, C. G.; C. Rossi, L. Tuttobello \& M. Ricci: The structure of Leucinostatin C, a minor peptide from Peacilomyces marquandii. Eur. J. Med. Chem. Chim. Ther. 21: $527 \sim 528,1986$

5) Rossi, C.; L. Tuttobello, M. Ricci, C. G. Casinovi \& L. RADICS: Leucinostatin D, a novel peptide antibiotic from Paecilomyces marquandii. J. Antibiotics 40: $130 \sim 133,1987$

6) Radics, L.; M. Kajtar-Peredy, C. G. Casinovi, C. Rossi, M. RicCi \& L. Tuttobello: Leucinostatins $\mathbf{H}$ and $\mathbf{K}$, two novel peptide antibiotics with tertiary amine-oxide terminal group from Paecilomyces marquandii. Isolation, structure and biologycal activity. J. Antibiotics 40: $714 \sim 716,1987$

7) Rossi, C.; M. Ricci, L. Tuttobello, S. Cerrini, A. Scatturin, G. Vertuani, V. Ambrogi \& L. Perioli: Leucinostatin F. A minor co-metabolite from Paecilomyces marquandii. Acta Technologiae et Legis Medicamenti 1: N.2, 109 112, 1990

8) Vertuani, G.; C. Falcomer, M. Boggian, G. Pochetti, S. Ceerini, M. Ricci, C. Rossi \& A. Scatturin: Structural studies of Leucinostatin A and its Boc-Aib-Leu-LeuAib-OMe tetrapeptide fragment. Int. J. Peptide Protein Res. 33: $162 \sim 170,1989$

9) Falcomer, C.; G. Vertuani, M. Boggian, G. Pochetti, S. Cerrini \& A. SCatturin: Conformational studies of synthetic Leucinostatin A fragments. In Second Forum on Peptides. Ed. A. Aubry et al. Vol. 174 pp. $383 \sim 386$, INSERM/J. Libbey Eurotext Ltd., London-Paris, 1989

10) Cerrini, S.; D. Lamba, A. Scatturin, C. Rossi \& C. UGHETTO: The crystal and molecular structure of the $\alpha$-helical nonapeptide antibiotic Leucinostatin A. Biopolymers 28: $409 \sim 420,1989$

11) Casinovi, C. G.; C. Rossi \& L. Tuttobello (Istitute Superiore di Sanità): A now peptide: Microbiologic procedures for its production and its uses as an antibiotic, cytotoxic and phytotoxic agent. Italian Pat. 47889A85, Mar. 28, 1985

12) Radics, L.; M. Kajtar-Peredy, M. Ricci \& C. Rossi: Leucinostatin A: High Field NMR Spectroscopy and Solution Conformation. To be published 